\title{
SUBSPACE BASED CHANNEL ESTIMATION WITH MULTIPLE RECEIVERS IN REUSE-1 OFDMA SYSTEMS - IMPACT ON DIVERSITY COMBINING AND NULLING
}

\author{
M. R. Raghavendra S. Bhashyam K. Giridhar \\ Telecommunication and Computer Networks Group, \\ Department of Electrical Engineering, \\ Indian Institute of Technology Madras, India
}

\begin{abstract}
In this paper, we evaluate the performance of subspace based channel estimation method with multiple receivers in reuse-1 OFDM systems. Users in reuse-1 cellular network will typically see signal to interference ratio (SIR) nearly $0 \mathrm{~dB}$ on the broadcast pilot subcarriers. Good channel estimation and tracking become very difficult for the interference limited users. The improved channel estimation method adopted in this paper assumes the multipath delay locations of the desired and the interferer channels do not overlap. Exploiting this information, it is possible to define a channel estimator/interpolator with a lower mean squared error compared to the conventional channel estimation techniques. The impact of the improved channel estimation method on the coded bit error rate (BER) performance of reuse-1 OFDM systems with receive diversity is presented through computer simulations.
\end{abstract}

\section{INTRODUCTION}

Orthogonal frequency division multiplexing (OFDM) has received considerable interest for its ability to mitigate frequency selective fading. OFDM based cellular systems are expected to be frequency reuse-1. The reuse-1 systems suffer from co-channel interference (CCI) which degrades the system performance significantly. In order to combat CCI for OFDM systems, antenna array based CCI suppression schemes are proposed in literature $[1,2,3]$. These techniques linearly combine different antenna outputs in order to estimate the transmitted information. The channel estimation plays an important role in defining the combiner. While [1] uses a minimum mean squared error (MMSE) based channel estimator (designed for fading channel without interference as in [7]), a pilot based channel estimation method capable of mitigating synchronous interference has been presented in [2]. This [2] assumes apriori knowledge of the multipath delays of desired and interferer signals in defining a channel estimator. Another pilot based MMSE (minimum mean square error) estimation method for time-varying channels with strong cochannel interference is presented in [3] for DVB-T (digital video broadcasting-terrestrial) systems. The estimator exploits the pseudorandom properties of the pilot sequences on interfering transmitters in mitigating co-channel interference. Since it models the interference components as white noise, it suffers from an irreducible error floor at high signal to noise ratios (SNR).

A subspace based channel estimation method for reuse-1 OFDM systems with strong co-channel interference is presented in [4]. The subspace based interference rejection method suppress the interferer

This work was supported by the National Research Fellowship Grant from IETE, India, on Emerging Wireless Technologies in the multipath-delay domain. This method assumes that the multipath delay locations of the desired and interferer channels do not exactly overlap. The non-overlapping nature of multipath delays corresponding to desired and interferer channels is plausible since the non-sample-spaced channel model is assumed. In this paper, we evaluate the performance of the above subspace based channel estimation technique with multiple receiver scenario. We observe that the high quality channel estimates results in significant BER improvement with multiple receivers.

\subsection{Basic Notation}

Bold face letters denote vectors or matrices; $(.)^{T},(.)^{*},(.)^{H}$ denote transpose, complex conjugate, Hermitian respectively; $E[$.$] denotes$ the expectation operator; $\mathcal{C N}(\mathbf{x}, \mathbf{C})$ represent complex Gaussian vector with mean $\mathbf{x}$ and variance $\mathbf{C}$; $\mathbf{I}_{K}$ denotes the $K \times K$ identity matrix; $\mathbf{0}_{p \times q}$ denotes the matrix of size $p \times q$ with zero entries; $\operatorname{diag}(\mathbf{x})$ is the diagonal matrix with elements of the vector $\mathbf{x}$ on its main diagonal; $\imath=\sqrt{-1}$.

\section{OFDM SYSTEM MODEL}

Consider an OFDM system operating with a bandwidth of $B=$ $\frac{1}{T} \mathrm{~Hz}$ ( $T$ is the sampling period). The system consists of $K$ subcarriers of which $K_{\mathrm{u}}$ are useful subcarriers (excluding guard bands and DC subcarrier) with the set $\mathcal{I}$ indicating useful subcarrier positions.

The baseband equivalent of the time-varying channel impulse response is modeled as a wide-sense stationary uncorrelated scatterer (WSSUS) zero mean complex Gaussian process. The channel impulse response has $L$ multipath components where each path is characterized by a complex gain factor $h_{l}$ and a delay $\tau_{l}$ [10], and has the form $h(\tau, t)=\sum_{l=0}^{L-1} h_{t}(l) \delta\left(\tau-\tau_{l}\right)$, where $h_{t}(m)$ is a zero-mean complex Gaussian random variable with $E\left[h_{t}(m) h_{t}^{*}(m)\right]=\sigma_{l}^{2}$ and $E\left[h_{t}(k) h_{t}^{*}(m)\right]=0$ for $k \neq m$. The paths fade independently according to the time-correlation function $E\left[h_{t}(l) h_{t^{\prime}}(l)\right]=$ $\sigma_{l}^{2} J_{0}\left(2 \pi f_{\mathrm{d}}\left(t-t^{\prime}\right)\right)$ where $J_{0}($.$) is the zeroth order Bessel func-$ tion of the first kind and $f_{\mathrm{d}}$ is Doppler frequency in Hz. The sampled channel in frequency domain is given as $\mathbf{H}_{n}=\mathbf{F} \mathbf{h}_{n}$ where $\mathbf{h}_{n}=\left[h_{n}(1), h_{n}(2), \ldots, h_{n}(L)\right]^{T}$ and the $(l, k)^{t h}$ element of the Fourier basis matrix $\mathbf{F}$ is

$$
[\mathbf{F}]_{l, k}=\exp \left(\frac{-\imath 2 \pi k \tau_{l}}{K T}\right) \quad k=0,1, \ldots, K-1, \quad l=1,2, \ldots, L .
$$

Assuming accurate frequency synchronization the received signal vector in frequency domain at time $n$ is,

$$
\mathbf{Y}_{n}=\mathbf{X}_{n} \mathbf{H}_{n}+\mathbf{V}_{n}
$$


where $\mathbf{X}_{n}$ is the diagonal matrix with data symbols $\left[X_{0, n}, X_{1, n} \ldots, X_{K-1, n}\right]$ and $\mathbf{H}_{n}$ is the sampled frequency response of the channel at $n^{\text {th }}$ OFDM symbol. The zero mean complex Gaussian noise vector $\mathbf{V}_{n}$ has distribution $\mathbf{V} \sim \mathcal{C N}\left(\mathbf{0}, \sigma^{2} \mathbf{I}_{K}\right)$.

Consider a downlink scenario with users at cell edge or sector edge. We assume the case of single transmit antenna at base station and two receive antennas for the mobile terminal. However, the method can be extended to systems with receive diversity. We restrict our discussion to a single interferer case, even though this method can be extended for multiple interferers as well. The user receives signals from both desired and interfering base stations (BS's). It is assumed that the receiver is synchronized to the desired BS and that all the base stations are frequency and frame synchronized. Let $\left\{\tau_{\mathrm{d}}\right\},\left\{\mathbf{h}_{\mathrm{d}, n}^{(1)}, \mathbf{h}_{\mathrm{d}, n}^{(2)}\right\}$ and $\left.\left\{\tau_{\mathrm{i}}\right\}, \mathbf{h}_{\mathrm{i}, n}^{(1)}, \mathbf{h}_{\mathrm{i}, n}^{(2)}\right\}$ denote the multipath delay locations (with $\tau_{\mathrm{d}, 1}=0$ ) and the channel impulse responses corresponding to desired and interferer base stations at the two receivers respectively. We assume that the two receive antennas experience independent fading. Let $L=L_{\mathrm{d}}+L_{\mathrm{i}}$ denote the total number of multipath components. We assume that the maximum delay spread of each of the channel (with respect to $\tau_{\mathrm{d}, 1}=0$ ) is smaller than the duration of cyclic prefix. Let $K_{\mathrm{d}}$ represent the number of subcarriers allocated to the desired user, with the set $\mathcal{I}_{\mathrm{d}}$ indicating their subcarrier indices. The received signal vector in frequency domain on the data subcarriers of the desired user at $n^{\text {th }}$ OFDM symbol is

$$
\begin{aligned}
& \mathbf{Y}_{n}^{(1)}=\mathbf{X}_{\mathrm{d}, n} \mathbf{H}_{\mathrm{d}, n}^{(1)}+\frac{1}{\sqrt{\gamma_{\mathrm{d}}}} \mathbf{X}_{\mathrm{i}, n} \mathbf{H}_{\mathrm{i}, n}^{(1)}+\mathbf{V}_{n}^{(1)} \\
& \mathbf{Y}_{n}^{(2)}=\mathbf{X}_{\mathrm{d}, n} \mathbf{H}_{\mathrm{d}, n}^{(2)}+\frac{1}{\sqrt{\gamma_{\mathrm{d}}}} \mathbf{X}_{\mathrm{i}, n} \mathbf{H}_{\mathrm{i}, n}^{(2)}+\mathbf{V}_{n}^{(2)}
\end{aligned}
$$

where $\gamma_{\mathrm{d}}$ represent signal to interference ratio (SIR) on data subcarriers.

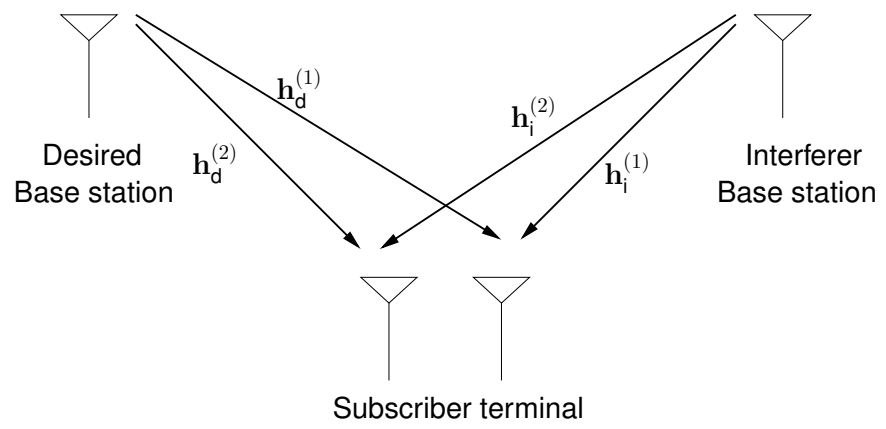

Fig. 1. OFDM system model

The OFDM frame structure is similar to the IEEE 802.16d/e WMAN standard [13] as captured follows. At the start of every OFDM frame, a preamble symbol is transmitted from all base-stations (BS). Assuming BS with a single transmit antenna, the three BS's (one per sector) are allocated orthogonal, equi-spaced subcarriers (i.e., preamble symbol is based on $1 / 3$ reuse design). Let $K_{\text {pre rep- }}$ resent the number of subcarriers allocated with the set $\mathcal{I}_{\text {pre }}$ indicating subcarrier positions for the desired transmitter. The following OFDM symbols contain pilot subcarriers in reuse-1 mode "shared" by all BS's for channel tracking. Let $K_{\text {pil }}$ represents the number of pilots with the set $\mathcal{I}_{\text {pil }}$ indicating their subcarrier indices. For a fixed number of pilot subcarriers in an OFDM symbol, it has been shown that the mean square error in the channel estimation is minimum if

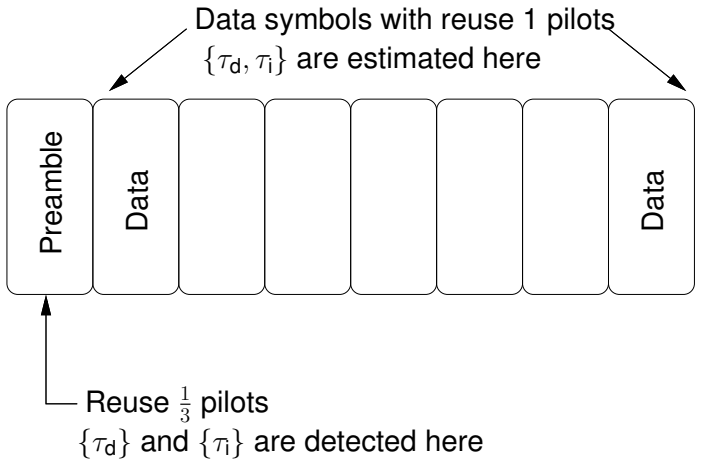

Fig. 2. OFDM frame structure

the pilots are equi-spaced and equi-powered on the frequency grid [9].

\section{CHANNEL ESTIMATION}

The channel estimation is carried out by inserting pilot subcarriers in the time-frequency grid. The estimates on the pilot subcarriers are then interpolated over data subcarriers. With reuse-1 OFDM, since the pilot subcarrier positions of the desired and interferer BS's overlap, the channel measured at the edge of the cell be severely corrupted. In order to combat interference on pilot carriers, the use of pseudo-random (PR) pilot sequences from the desired and interferer BS's is proposed in $[13,14]$. While this gives some co-channel interference (CCI) mitigation ${ }^{1}$, such schemes fail when interference is strong. In further discussions we refer the above channel estimation technique as PR-mls (i.e., using modified least squares based technique [8] with pseudo-random pilots to combat interference).

In this paper, we have adopted the channel estimation technique as proposed in [4]. The pilot sequences from desired and interferer BS's are made same so as to measure the statistics of the combined channel. From the estimated statistics, the proposed method computes the multipath delays of both the desired and interfering channels at the receiver. Exploiting this information, the interferer is suppressed in multipath-delay domain (the domain where the multiple paths can be resolved independently) and the improved channel interpolator is defined. The technique is briefly discussed in subsequent sections.

\subsection{Estimating and tagging multipath delays}

The least squares (LS) channel estimates on pilot subcarriers [8] on the $j^{\text {th }}$ antenna is given by

$$
\begin{aligned}
\widehat{\mathbf{H}}_{n}^{(j)} & =\left(\overline{\mathbf{X}}_{n}^{(j)}\right)^{-1} \overline{\mathbf{Y}}_{n}^{(j)}=\overline{\mathbf{H}}_{\mathrm{d}, n}^{(j)}+\left(1 / \sqrt{\gamma_{\mathrm{p}}}\right) \overline{\mathbf{H}}_{\mathrm{i}, n}^{(j)}+\left(\overline{\mathbf{X}}_{\mathrm{d}, n}^{(j)}\right)^{-1} \overline{\mathbf{V}}_{n}^{\prime} \\
& =\overline{\mathbf{F}}_{\mathrm{d}} \mathbf{h}_{\mathrm{d}, n}^{(j)}+\left(1 / \sqrt{\gamma_{\mathrm{p}}}\right) \overline{\mathbf{F}}_{\mathrm{i}} \mathbf{h}_{\mathrm{i}, n}^{(j)}+\underbrace{\left(\overline{\mathbf{X}}_{\mathrm{d}, n}^{(j)}\right)^{-1} \overline{\mathbf{V}}_{n}^{\prime}}_{\overline{\mathbf{V}}_{n}^{\prime} \sim \mathcal{C N}\left(\mathbf{0}, \sigma^{2} \mathbf{I}_{K_{\mathrm{pil}}}\right)}
\end{aligned}
$$

${ }^{1} \mathrm{CCI}$ mitigation on channel estimates is possible provided the CCI is weak and/or the number of pilots is much larger than channel impulse response length 
where $\overline{\mathbf{F}}_{\mathrm{d}}, \overline{\mathbf{F}}_{\mathrm{i}}$ are sub-matrices derived by retaining rows corresponding to pilot subcarrier locations in $\mathbf{F}_{\mathrm{d}}, \mathbf{F}_{\mathrm{i}}$. The signal-space spanned by the pilot channel estimates exhibits this shift-invariance property enabling ESPRIT for multipath delay estimation ${ }^{2}$. The autocorrelation matrix $\widehat{\mathbf{R}}_{\mathrm{H}}$ of channel estimates is estimated as

$$
\widehat{\mathbf{R}}_{\mathrm{H}}=\sum_{m=1}^{2} \sum_{n=1}^{M} \widehat{\mathbf{H}}_{n}^{(m)}\left(\widehat{\mathbf{H}}_{n}^{(m)}\right)^{H}
$$

where $M$ is the number of OFDM symbols considered for averaging. The number of paths are estimated as the number of dominant eigenvalues $(\widehat{L})$ of $\widehat{\mathbf{R}}_{\mathrm{H}}$ as in [10]. The eigenvectors corresponding to $\widehat{L}$ dominant eigenvalues forms the delay-subspace basis $\widehat{\mathbf{U}}_{\mathrm{s}}$. Let $\widehat{\mathbf{U}}_{1}$ and $\widehat{\mathbf{U}}_{2}$ represent the shift invariant subspaces derived from $\widehat{\mathbf{U}}_{\mathrm{s}}$. The placement of zero-tones (guard carriers) decides the choice of $\widehat{\mathbf{U}}_{1}$ and $\widehat{\mathbf{U}}_{2}$ from $\widehat{\mathbf{U}}_{\mathrm{s}}$. Exploiting the shift invariance structure of the subspaces $\widehat{\mathbf{U}}_{1}$ and $\widehat{\mathbf{U}}_{2}$, the multipath delays are estimated as [11] $\widehat{\tau}_{l}=\arg \left(\phi_{l}^{*}\right) K T /(2 \pi P)$ for $l=0,1, \ldots, \widehat{L}-1$ where $P$ denote the shift (in subcarriers) between the subspaces $\widehat{\mathbf{U}}_{1}$ and $\widehat{\mathbf{U}}_{2}, \arg \left(\phi_{l}^{*}\right)$ denotes the phase angle (in the range $[0,2 \pi)$ ) of $\phi_{l}^{*}$ and $\left\{\phi_{l}\right\}_{l=0}^{l=\widehat{L}-1}$ are the eigenvalues of the matrix $\psi=\left(\widehat{\mathbf{U}}_{1}^{H} \widehat{\mathbf{U}}_{1}\right)^{-1} \widehat{\mathbf{U}}_{1}^{H} \widehat{\mathbf{U}}_{2}$.

The "interference free" channel estimates derived from the preamble corresponding to the desired BS are projected onto the combined basis derived from the estimated multipath delays $\left\{\widehat{\tau}_{l}\right\}_{l=0}^{\widehat{L}-1}$. The contribution along different basis vectors are then used to separate the multipath delays corresponding to the desired channel.

Let $\widehat{\mathbf{H}}_{\mathrm{p}}$, a $K_{\text {pre }} \times 1$ vector, represent the "interference free" channel estimates corresponding to desired transmitter derived from the preamble symbol. The interference free channel estimates $\widehat{\mathbf{H}}^{\mathrm{p}}$ are projected onto the combined Fourier basis derived from the estimated multipath delays as,

$$
\widehat{\mathbf{h}}^{(j)}=\left(\widetilde{\mathbf{F}}_{\mathrm{p}}^{H} \widetilde{\mathbf{F}}_{\mathrm{p}}\right)^{-1} \widetilde{\mathbf{F}}_{\mathrm{p}}^{H} \widehat{\mathbf{H}}_{\mathrm{p}}^{(j)}
$$

where $\widetilde{\mathbf{F}}_{\mathrm{p}}$ is $K_{\text {pre }} \times \widehat{L}$ matrix with $\left[\widetilde{\mathbf{F}}_{\mathrm{p}}\right]_{k, l}=\exp \left(-\frac{j 2 \pi \widehat{\tau}_{l} \mathcal{I}_{\text {pre }}(k)}{K T}\right)$ for $k=1,2, \ldots, K_{\text {pre }}$ and $l=1,2, \ldots, \widehat{L}$. The vector $\widehat{\mathbf{h}}^{(j)}$ represents the components of $\widehat{\mathbf{H}}_{\mathrm{p}}^{(j)}$ along different basis-vectors associated with multipath delays. The projections $\frac{\left|\widehat{\mathbf{h}}^{(1)}\right|^{2}+\left|\widehat{\mathbf{h}}^{(2)}\right|^{2}}{2}$ are then compared with scaled noise variance $\kappa \sigma^{2}$ and the components that exceed the scaled noise variance are counted as dominant. Let $\mathcal{T}_{\mathrm{d}}$ (a $\widehat{L}_{\mathrm{d}} \times 1$ vector) denote the indices of multipath delays corresponding to dominant projections.

\subsection{Interference rejection and channel interpolation}

Once the multipath delay locations of the desired channel are known, the delay-domain channel gains corresponding to $j^{\text {th }}$ receive antenna are estimated by projecting the pilot channel estimates onto the Fourier basis as follows:

$$
\widehat{\mathbf{h}}_{\mathrm{c}, n}^{(j)}=\left(\widetilde{\overline{\mathbf{F}}}_{\mathrm{c}} \widetilde{\overline{\mathbf{F}}}_{\mathrm{c}}\right)^{-1} \widetilde{\overline{\mathbf{F}}}_{\mathrm{c}} \widehat{\overline{\mathbf{H}}}_{n}^{(j)}
$$

where $\widetilde{\overline{\mathbf{F}}}_{\mathrm{c}}$ is $K_{\text {pil }} \times \widehat{L}$ matrix with $\left[\widetilde{\overline{\mathbf{F}}}_{\mathrm{c}}\right]_{k, l}=\exp \left(-\frac{\imath 2 \pi \widehat{\tau}_{l} \mathcal{I}_{\text {pil }}(k)}{K T}\right)$ for $k=1,2, \ldots, K_{\text {pil }}$ and $l=1,2, \ldots, \widehat{L}$. The desired channel impulse response estimates are the components of $\widehat{\mathbf{h}}_{\mathrm{c}, n}^{(j)}$ with indices

\footnotetext{
${ }^{2}$ The presence of zero-tones should be accounted in deriving shift invariant subspaces from the signal-space of the channel estimates
}

$\mathcal{T}_{\mathrm{d}}$. Finally, we derive frequency domain channel estimates on the subcarriers of the desired user for the $j^{\text {th }}$ antenna as

$$
\widehat{\mathbf{H}}_{\mathrm{d}, n}^{(j)}=\widetilde{\mathbf{F}}_{\mathrm{d}} \widehat{\mathbf{h}}_{\mathrm{d}, n}^{(j)}
$$

where $\widetilde{\mathbf{F}}_{\mathrm{d}}$ is a $K_{\mathrm{d}} \times \widehat{L}_{\mathrm{d}}$ matrix with $\left[\widetilde{\mathbf{F}}_{\mathrm{d}}\right]_{k, l}=\exp \left(-\frac{\imath 2 \pi \widehat{\tau}_{l} \mathcal{I}_{\mathrm{d}}(k)}{K T}\right)$ for $k=1,2, \ldots, K_{\mathrm{d}}$ and $l \in \mathcal{T}_{\mathrm{d}}$. The channel estimates $\widehat{\mathbf{H}}_{\mathrm{d}, n}$ can be further improved by exploiting the time correlation of temporal channel estimates $\widehat{\mathbf{h}}_{\mathrm{d}, n}$ due to Doppler using a FIR (finite impulse response) filter. The co-efficients of the FIR filter are derived using Weiner filter theory [7].

\section{SYMBOL DETECTION WITH MULTIPLE RECEIVERS}

In this section we consider three symbol detection techniques used for systems with antenna arrays.

\subsection{Diversity Combining techniques}

\subsubsection{Maximum ratio combining}

In MRC, the received signals from all antennas are weighted and combined to maximize the instantaneous signal to noise ratio (SNR) at the receiver [5]. The MRC is the optimal combining scheme in the absence of co-channel interference. The receiver combining for two branches is,

$$
\widehat{X}_{\mathrm{d}, n}(k)=\frac{Y_{n}^{(1)}(k) H_{\mathrm{d}, n}^{(1)}(k)+Y_{n}^{(2)}(k) H_{\mathrm{d}, n}^{(2)}(k)}{\left|H_{\mathrm{d}, n}^{(1)}(k)\right|^{2}+\left|H_{n}^{(2)}(k)\right|^{2}}
$$

where $\widehat{X}_{\mathrm{d}, n}(k)$ is an estimate of the transmitted symbol $X_{\mathrm{d}, n}(k)$. Observe that the MRC needs channel state information of desired channel at receiver.

\subsubsection{Minimum mean squared error-diversity combiner (MMSE-} $D C)$

The diversity combiner that reduces the mean squared error between the transmitted and the estimated symbol and takes the form [7, 6]

$$
\widehat{X}_{\mathrm{d}, n}(k)=\mathbf{w}_{n}(k)^{H} \mathbf{Y}_{n}(k)
$$

where $\mathbf{Y}_{n}(k)=\left[Y_{n}^{(1)}(k) Y_{n}^{(2)}(k)\right]^{T}$ and the weight vector $\mathbf{w}_{n}(k)$ is derived as

$$
\mathbf{w}_{n}(k)=\left(\mathbf{R}_{n}(k)+\gamma \mathbf{I}\right)^{-1} \mathbf{H}_{\mathrm{d}, n}(k)
$$

with $\mathbf{H}_{\mathrm{d}, n}(k)=\left[H_{\mathrm{d}, n}^{(1)}(k) H_{\mathrm{d}, n}^{(2)}(k)\right]^{T}$ and $\mathbf{R}_{n}(k)=E\left[\mathbf{Y}_{n}(k) \mathbf{Y}_{n}(k)^{H}\right]$. Observe that the MMSE-DC needs desired channel information and correlation statistics of the received signals. It is to be noted that in the absence of interference the MRC and MMSE-DC are equivalent.

\subsection{Nulling}

In order to combat interference with multiple receivers, a nulling (zero forcing) solution to suppress the interference can be derived. The set of equations on the $k^{\text {th }}$ subcarrier in (3), (4) can be written as

$$
\mathbf{Y}_{n}(k)=\mathbf{H}_{n}(k) \mathbf{X}_{n}(k)+\mathbf{V}_{n}(k)
$$


where $\mathbf{H}_{n}(k)=\left[\begin{array}{cc}H_{\mathrm{d}, n}^{(1)}(k) & H_{\mathrm{i}, n}^{(1)}(k) \\ H_{\mathrm{d}, n}^{(2)}(k) & H_{\mathrm{i}, n}^{(2)}(k)\end{array}\right], \mathbf{X}_{n}(k)=\left[\begin{array}{c}X_{\mathrm{d}, n}(k) \\ X_{\mathrm{i}, n}(k)\end{array}\right]$ and $\mathbf{V}_{n}(k)=\left[\begin{array}{c}V_{n}^{(1)}(k) \\ V_{n}^{(2)}(k)\end{array}\right]$.

An equalizer is defined to null the effect of interference at the receiver, i.e, we design an equalizer $\mathbf{G}_{n}(k)$ such that

$$
\mathbf{G}_{n}^{H}(k) \mathbf{H}_{n}(k)=\left[\begin{array}{ll}
1 & 0
\end{array}\right]
$$

and an estimate of the desired symbol is given by

$$
\widehat{X}_{\mathrm{d}, n}(k)=\mathbf{G}_{n}^{H}(k) \mathbf{Y}_{n}(k) .
$$

Observe that defining a nuller need channel information of both desired and interferer channels.

\section{SIMULATION AND RESULTS}

We demonstrate the performance of the proposed estimator by computer simulations. An OFDM system is simulated with following parameters [13]: center frequency $f_{\mathrm{c}}=2.2 \mathrm{GHz}$, bandwidth $B=$ $\frac{1}{T}=5 \mathrm{MHz}, K=512, K_{\mathrm{u}}=420, L_{\mathrm{cp}}=32$, OFDM symbol duration $T_{\mathrm{s}}=\left(K+L_{\mathrm{cp}}\right) T, K_{\mathrm{pre}}=\frac{420}{3}=140, K_{\mathrm{pil}}=53, K_{\mathrm{d}}=50$. A OFDM burst consists of 5 frames and each frame consists of 100 OFDM symbols. A preamble symbol is transmitted at the start of every frame. We have considered a 4 tap channel model for the desired and interferer signals. The power delay profile (pdp) of the channel is assumed to be uniform. The channel paths fade independently according to Jakes' power spectrum [12]. The normalized fade rate is $f_{\mathrm{d}} T_{\mathrm{s}}=0.004$.

The performance of the algorithm is evaluated using channel mean square error (MSE) and bit error rate (BER) by averaging over channels with different multipath delay locations. In evaluating BER results, we consider coded OFDM system. The input bits are encoded with rate $1 / 2$ parallel concatenated convolutional codes (turbo-codes) and then modulated using QPSK scheme. The synchronous interferer signal is also assumed to be QPSK modulated. The performance of the system is evaluated by setting the SIR on pilot subcarriers $\gamma_{\mathrm{p}}=0 \mathrm{~dB}$. The MSE is defined as (for some $n$ )

$$
\mathrm{MSE}=\frac{1}{K_{\mathrm{d}}} \sum_{k \in \mathcal{I}_{\mathrm{d}}} E\left\{\left|H_{\mathrm{d}, n}^{(1)}(k)-\widehat{H}_{\mathrm{d}, n}^{(1)}(k)\right|^{2}\right\} .
$$

Fig. 3 compares the MSE performance of subspace (SS) method [4] and pseudo-random (PR-mls) pilot data based channel estimation methods with co-channel interference. The MSE is measured at the end of $n=40$ OFDM symbol. It is observed that the PR-mls based channel estimation method fails for severe CCI and suffers from an irreducible error floor.

Fig. 4 compare the coded BER performance of PR-mls based channel estimation technique for different data SIRs. We have compared the performance of three detection techniques discussed in section 4. Since the PR-mls based channel estimates are poor in MSE the BER suffers from irreducible error floor at higher SNRs for all data SIRs.

Fig. 5 compare the coded BER performance of the proposed subspace (SS) based channel estimation technique for different data SIRs and different symbol detection techniques. The performance of the diversity combining symbol detection techniques (MRC, MMSEDC) improves with higher data SIRs. Note that the nulling (zeroforcing solution) based detection does not exploit the receive diversity and hence it suffers from performance loss when compared to

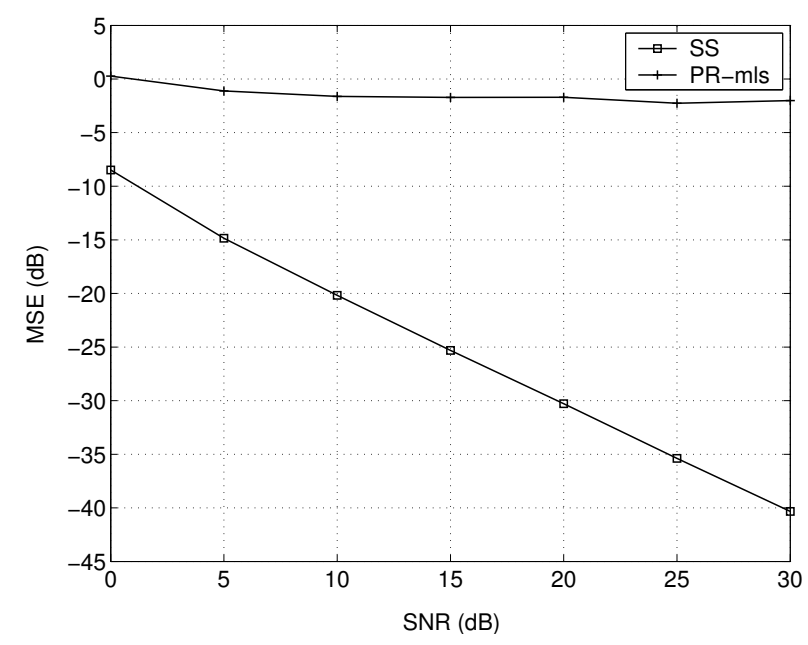

Fig. 3. MSE convergence plot comparing proposed subspace method (SS) with PR-mls based method

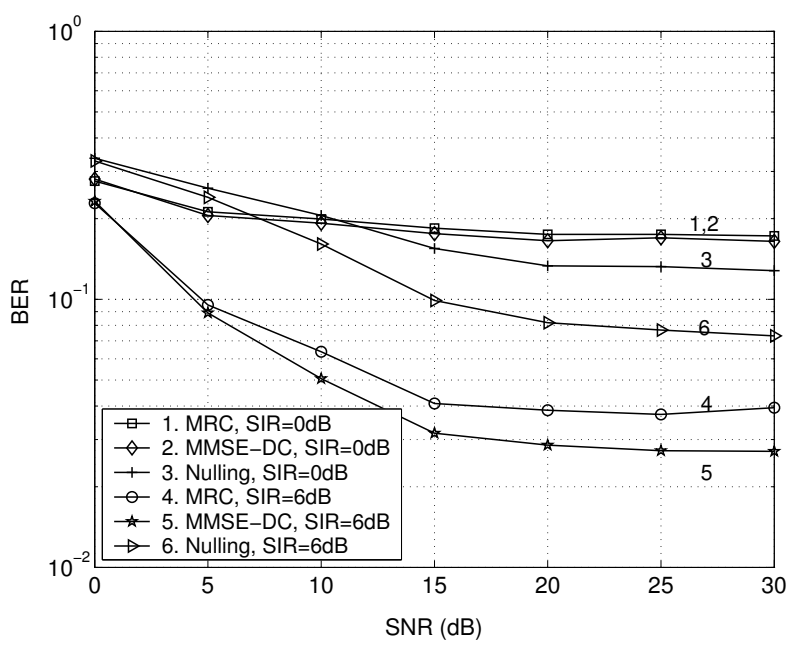

Fig. 4. Coded BER performance of the PR-mls method for different $\gamma_{\mathrm{d}}$

diversity combing techniques at higher SIRs. However, observe that the performance of the nuller improves with SNR at all SIRs since it can completely null the interferer signal.

\section{CONCLUSION}

The performance of subspace based channel estimation method with multiple receivers in reuse-1 OFDM systems is presented. The subspace based channel estimation method reject the interferer in the multipath-delay domain. With the subspace based channel estimation method it is possible to estimate and track both the desired and interferer channels at the receiver. The simulation results provided reveals that an improved channel estimation plays an important role in symbol detection for interference limited systems. 


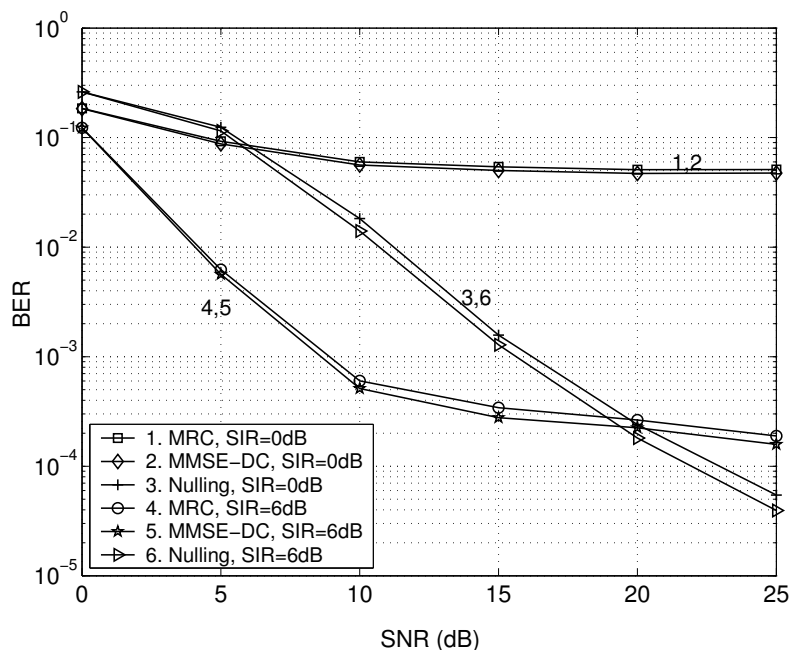

Fig. 5. Coded BER performance of the proposed subspace method for different $\gamma_{\mathrm{d}}$

\section{REFERENCES}

[1] Ye Li and N. R. Solenberger, "Adaptive antenna arrays for OFDM systems with co-channel interference"IEEE Transactions on Communications, vol. 47, no. 2, pp. 217-229, February 1999.

[2] A. Jeremic, T. A. Thomas and A. Nehorai, "OFDM channels estimation in the presence of interference" IEEE Trans. on Signal Processing, vol. 52, no. 12, 3429-3439, December 2004.

[3] S. Dieter, G. Matz, H. Franz and P. Loubaton, "MMSE estimation of time varying channels for DVB-T systems with strong co-channel interference" Invited paper in Proceedings of EUSIPCO-2002, September 2002.

[4] M. R. Raghavendra, S. Bhashyam, K. Giridhar, " Parametric channel estimation in reuse-1 OFDM systems," accepted in IEEE International Conference on Communication (ICC), 2007.

[5] M. Z. Win, and J. H. Winters, "Analysis of hybrid selection/maximal-ratio combining in Rayleigh fading," In Proc. of IEEE ICC 1999, Vol. 1, pp. 6-10, 1999.

[6] G. Luca, A. Luici, P. Giovanni, O. Shuktai, M. Okada, and K. Shozo, "Co-channel interference cancellation based on MIMO-OFDM systems," IEEE Wireless Communications Magazine, pp. 8-17, December 2002.

[7] Y. Li, L. J. Cimini Jr., and N. R. Sollenberger, "Robust channel estimation for OFDM systems with rapid dispersive fading channels," IEEE Trans. on Communications, vol. 46, no. 7, pp. 902-915, July 1998.

[8] J. J. van de Beek, O. Edfors, M. Sandell, S. K. Wilson, and P. O. Borjesson, "On channel estimation in OFDM systems," in Proceedings of IEEE VTC'1995, vol.2, pp. 815-819, July 1995.

[9] S. Ohno and G. B. Giannakis, "Capacity maximizing MMSEoptimal pilots for wireless OFDM over frequency-selective block Rayleigh-fading channels," IEEE Trans. on Information Theory, vol. 50, no. 9, pp. 2138-2145, September 2004.

[10] M. R. Raghavendra, S. Bhashyam, and K. Giridhar, "Exploiting hopping pilots for parametric channel estimation in OFDM systems", IEEE Signal Processing Letters, vol.12, no. 11, pp. 737-740, November 2005.

[11] R. Roy and T. Kailath, "ESPRIT - Estimation of signal parameters via rotational invariance techniques," IEEE Trans. on Acoustics, Speech, and Signal Processing, vol. 37, no. 7, pp. 984-995, July 1989.

[12] W. C. Jakes, Microwave mobile communications, IEEE Press, 1993.

[13] IEEE P802.16-2004, Standard for local and metropolitan area networks Part 16: Air interface for fixed broadband wireless access systems, 2004.

[14] U. Reimers, "Digital video broadcasting," IEEE Communications Magazine, pp. 104-110, June 1998. 